\title{
Virtual Construction Kits
}

\author{
Nikolay Selivanov \\ Studio of art designing, Moscow
}

\begin{abstract}
The "Studio of art designing" develops and publishes electronic creative education programs for children and young people. All virtual kits represent miniature programs each designed for a small but clear task. The main purpose of each construction kit is to reveal the meaning of some aspect of the creative process. Virtual construction kits suggest the user making their own work of art. However, this creative process is done with the help of means and methods that develop the user's thinking. Thus, the user gets to know this phenomenon "from the inside." Virtual kits have found application in textbooks for a comprehensive school and as an accompaniment to museum projects. Projects related to the Russian Avant-Garde, antique vase-painting, steampunk, infographics, animation, "laterna magica" and visual storytelling are used in many secondary schools and museums in Russia.
\end{abstract}

Keywords: electronic creative education, virtual construction kits, art education, creative thinking, artistic thinking

\section{Introduction}

The art of the past is the thing in itself. How to work the way through these borders and discover an opportunity to perceive the visual heritage, to turn hidden and unnecessary into the actual and essential? Virtual construction sets, which will be discussed below, were created as an answer to this question. A diverse range of projects for the implementation of Information and Communication Technology (ICT) in higher and secondary education, museum projects, art and curatorial projects in the field of new media led me to this form of digital tools.

I refer virtual construction sets to the class of tools designed for creative modeling of new images but limited to a given framework of visual and semantic contexts. In my teaching practice, this class of instruments also includes museum role-playing and board games. Such tools are aimed at the active perception of the semantic content of culture within the process of heritage interpretation. After all, works that are easily created by users of virtual construction sets become part of their reality, they directly affect their picture of the world and begin to control their behavior (Korzybski, 1995). Once abstracted into various representational forms, reality returns to the process of actual thinking, bringing with it a meaningful cultural experience.

It is in this sense that we can say that the development and use of virtual construction sets were aimed at the practical solution of visual literacy issues. I see construction sets as effective means of revealing the perception of artistic heritage, which operates on a pre-verbal level. Here we can recall Buhler's hypothesis about pre-speech instrumental thinking, about "subjectively meaningful, that is, consciously purposeful activity" Buhler, 1924, p.100), which in this case is stimulated and carried out with the help of virtual construction sets.

I define the functionality of virtual construction sets as the action of "second-level stimuli" (Vygotsky, 1984, p.503). That is, the use of virtual construction sets is aimed at establishing a connection between a sign and its meaning in the process of combinatorial activity with visual elements that stimulate and direct the thinking process. The result is the creation of a new image and a new semantic situation, which manifests itself in the context of the cultural context set by the design. In this way, a connection is established for the perception of the visual heritage.

The characteristics of the given examples of virtual construction sets by their goals, topics, and recommended age are placed at the end of the text. 
The emergence of the idea and the development of the concept of virtual construction sets An idea of the virtual construction kit is associated with undertaking one project. The project was aimed at holding a workshop for secondary school students, without any specific artistic skills. The creative task was connected with an intricate genre of contemporary art - artist's book. Artist's book was positioned as a complex media nonlinear hybrid. The teenagers were asked to create an object that would combine text, color, pictograms and illustrations into a single structure. It was no less important to come up with a special sequence of "reading" this object.

Figure 1

Virtual construction kit. "Artist's book", 2004

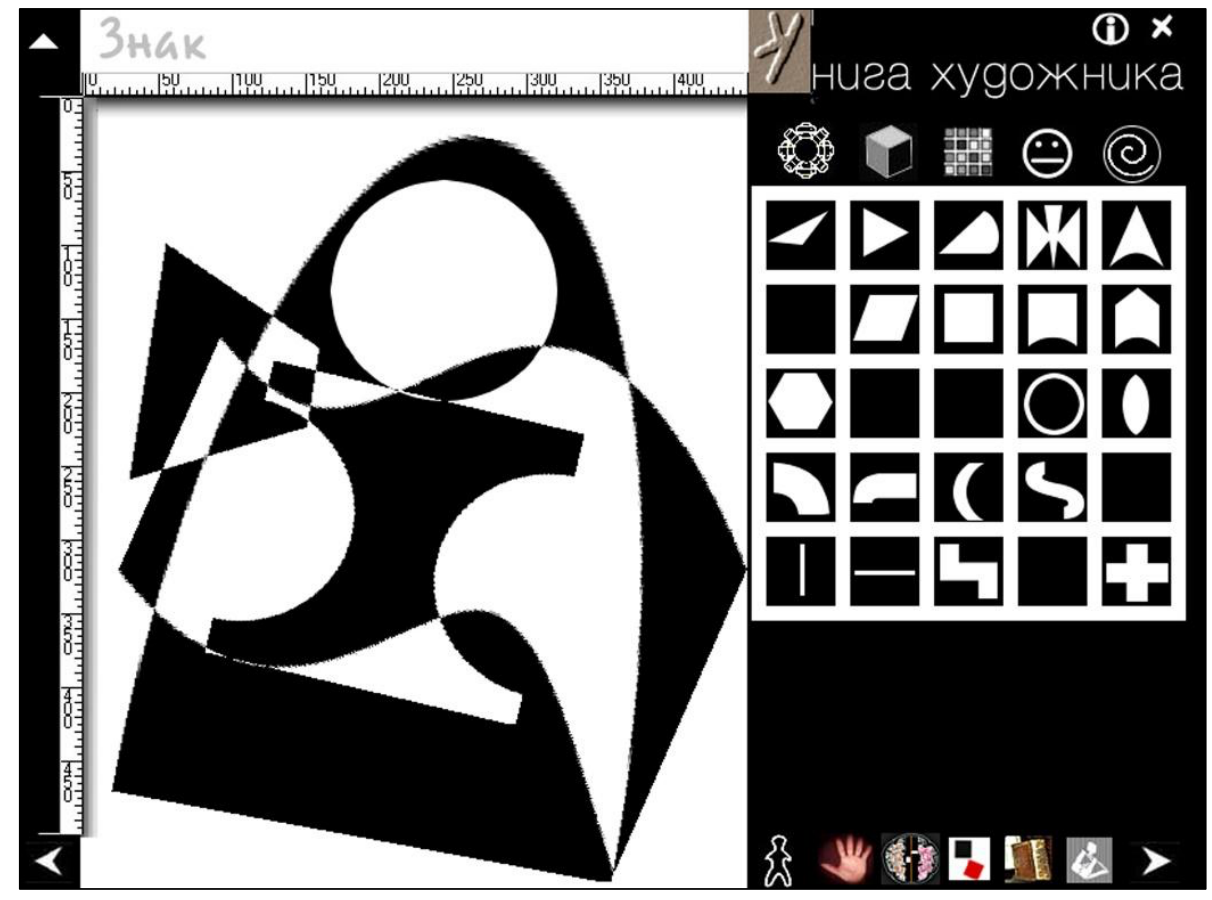

The large-scale participation of this workshop and time limits forced me to invent the way to manage the creative educational process. I have prepared a presentation and tried to explain the task and describe the possibilities for constructing such an object using a construction kit. At that time, I was a frequent user of the Macromedia Director program and benefited from its capabilities to create unusual interactive presentations. Then, I made several construction kits for the workshop, with the help of which I was able to explain the main steps of the proposed task.

Figure 2

Pictograms created with the virtual construction kit. "Artist's Book", 2004



The set of these first construction kits consisted of the sign, form and color construction kits. The sign construction kit was especially interesting, and l've been constantly using it in my work with children since 
then. With this construction kit, I was able to see the possibilities of this construction kit that allowed me to see the possibilities of the development of children's brain building. As shown in Figure 1, I made a small set of parts of an unusual shape for it; moreover, each of the parts was in a single copy which led to a limitation of design possibilities. This limitation has proven to be a very effective way of stimulating creative imagination and combinatorial thinking. All the details in the kit are random. These are irregular geometric shapes or polyhedrons, which resemble the scraps of paper after collage work. Angular, curved shapes do not evoke any associations, do not resemble any specific items or details. While creating these elements I aimed precisely on ensuring that they did not orient the students' imaginations in any particular direction. The purpose of a kit of such shapes is to create the basis for unexpected visual discovery. Another goal in using random shapes is the impossibility of creating a naturalistic image. The result is always expressive, but conditional and rather abstract (Figure 2). The graphic expressiveness and uniqueness of the images created with the help of this sign construction kit is the result of a special effect - the inversion of the colors and the forms when they are superimposed on each other. All parts of the construction kit are initially black, but when their parts hit each other, these parts turn white. Such an inversion can be used as a special visual technique with multiple superimpositions of silhouettes on top of each other (Figure 1).

Unfortunately, the electronic application "Artist's Book" was created a while ago, when the standard screen resolution was very low. It was not possible to create complex graphic works on a small working tablet of the construction kit. Parts of this constructor can only be scaled and rotated.

\section{Figure 3}

Stepan Ageev. "Artist's book". Academic work, 2016

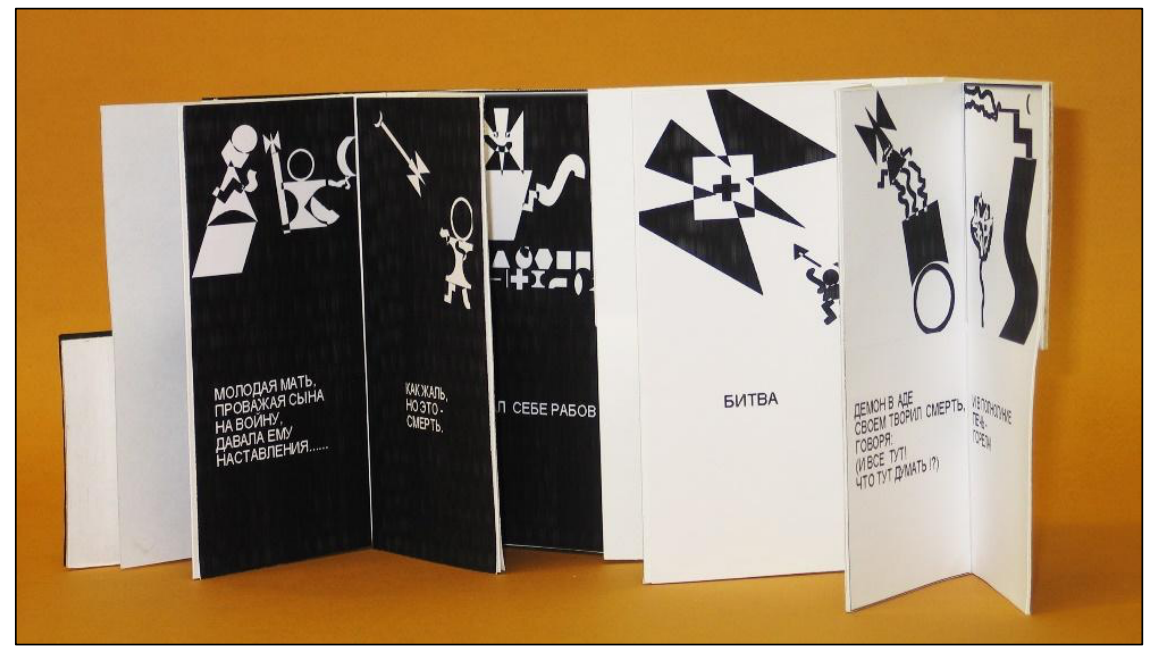

Students are always involved in the tasks associated with this construction kit with interest and enthusiasm, because it becomes a challenge for them and engages them actively. I realized that the virtual construction kit is an effective tool for creating the very possibilities of thinking that Vygotsky defined as "psychological tools" (Vygotsky, 1984) - tools directed inward to the development of their thinking. I define virtual construction kits in the same way - these are digital tools that form psychological tools.

The electronic system "Artist's Book," which includes these virtual construction kits, remains relevant for our activities with children, in which questions of creative work with information, the design of icons, and the creation of visual narratives are raised. Working with construction kits from the "Artist's Book" is also possible for young children. Therefore, often the first acquaintance with the computer, as a creative tool, we carry out with the help of the "Artist's Book."

Another important aspect that was revealed in the "Artist's Book" is an ability to create real objects using these construction kits. Thus, with the help of the color construction kits, students select necessary colors for their projects, with the help of the form construction kits, they think over the shape of the object and the 
sequence of its disclosure, and with the help of the sign kit they create graphic content. Graphic images can be printed, and you can create a real object - an artist's book (Figure 3). But in the "Artist's Book" there was not yet a module for working with text, so the text is created in any graphic editor. Later, when preparing the electronic system "Artist's Book" for publication, I supplemented the set of construction kits with one more, with the game purpose. This is a construction kit called "Dump" (Figure 4), in which the details of all the construction kits are mixed and there is an opportunity to use color, and I made some of the details move. It seemed to me necessary to supplement the set of construction kits with one more - providing the possibility of free action. The fact is that simultaneously with the work on these constructors, I carried out my first game museum project. The theme of the game, as the territory of creative freedom necessary for design, influenced all subsequent developments of virtual construction kits.

\section{Figure 4}

Virtual construction "Dump". "Artist's book", 2004

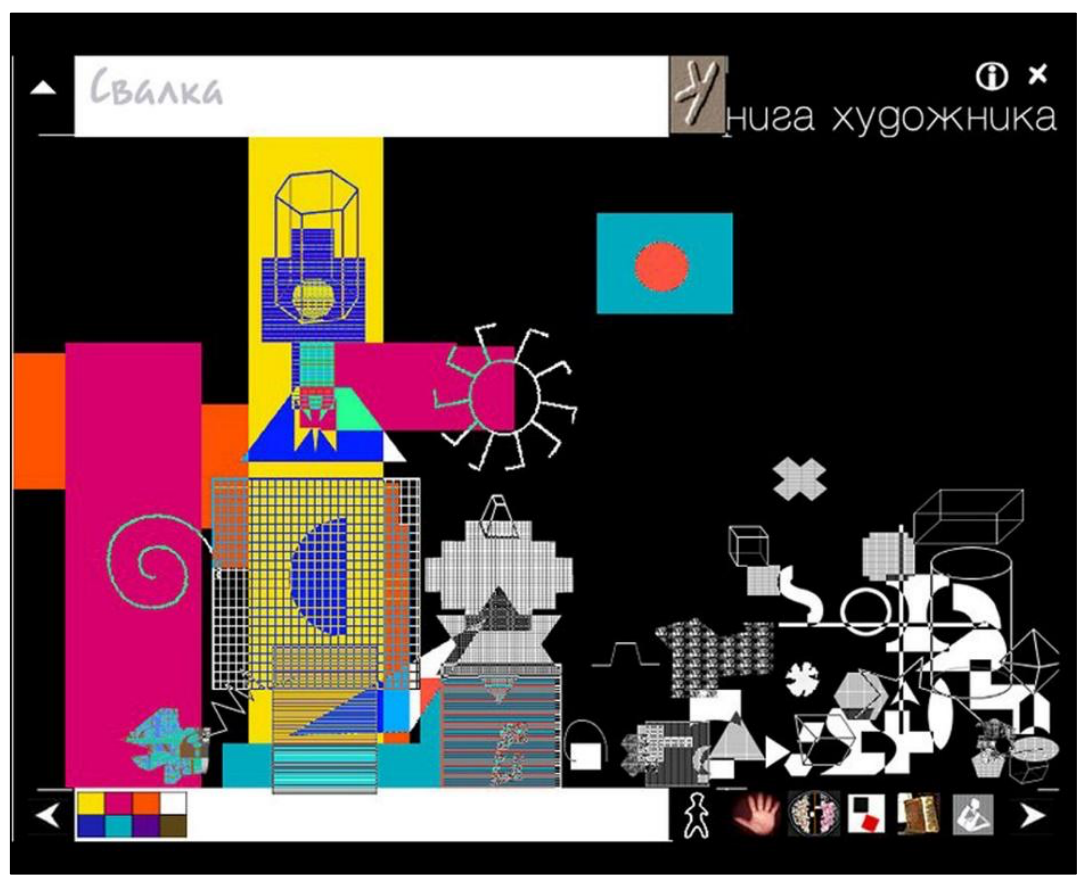

The idea to use the construction kit method to issue an assignment for the Workshop of the Artist's Book was naturally not accidental. Back in the mid-nineties, I was struck by the acquaintance with the outstanding pedagogical experience - the construction kit "Gifts" by the Austrian teacher of the first half of the 19th century Friedrich Fröbel.

Friedrich Fröbel (1838) suggested the usage of construction kits to develop child's thinking (Brosteman, 1997). But since then, I have been developing these ideas in several directions, one of which is virtual construction kits. I define the kit by Friedrich Fröbel as a construction kit of the eidetic type (from Eidos), aimed at the developing of sign-symbolic representations of the child with the help of abstract forms that the adult gives meaning to during the game (Figure 5).

In virtual construction kits, myself as a developer, play the role of an adult. In this sense, my task is complicated by the fact that I cannot communicate with a child using the virtual construction kit directly. Therefore, my project task for developing kits includes not only creating a set of elements, but also creating a semantic context that determines the content of the game with a construction kit. This context in the mind of a child is formed in two ways - by a small description and, most importantly, by means of visual design. 


\section{Figure 5}

The explanation of task meaning with aid of the construction kit Gifts by Friedrich Fröbel.

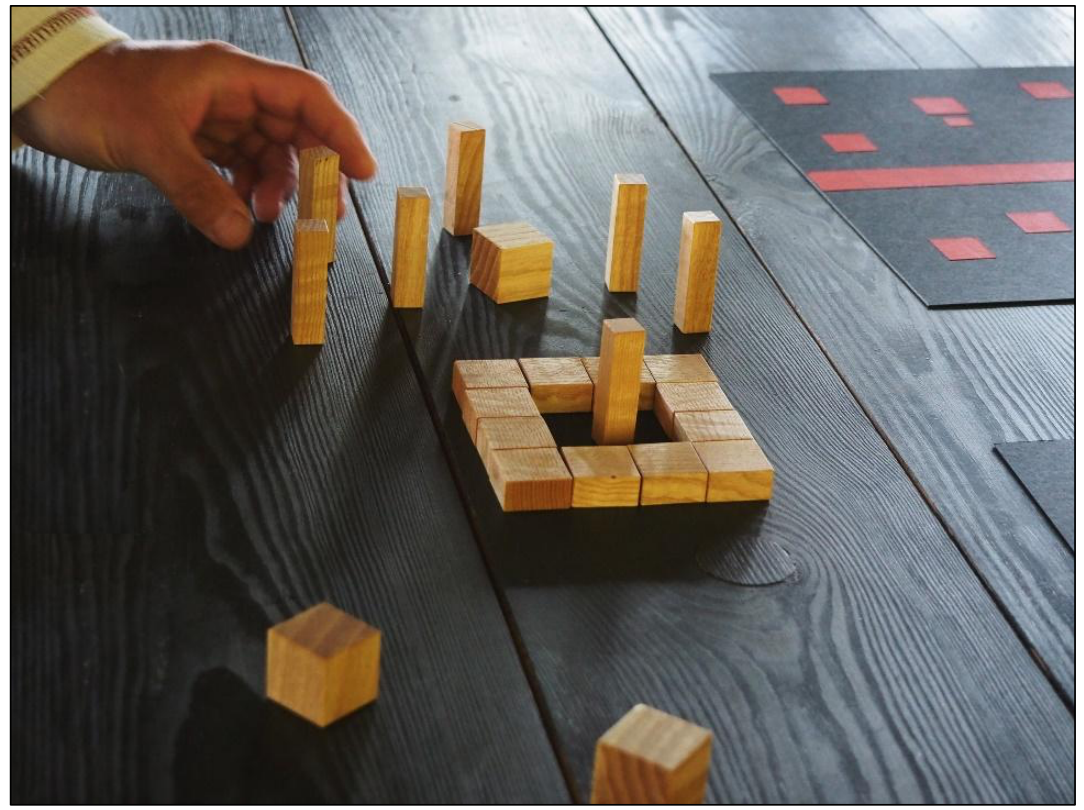

The next virtual construction kit became part of a large research project called "The Return of the Magi" (Figure 6), which I carried out together with my student Olga Rozmakhova, a medievalist. This was the first experience of creating a semantic construction kit. We wanted to make a magical game with precious decorations of the most famous medieval relics. The set of details for the kit was to represent the semantics of the objects used at the Christian liturgy. But we have not achieved this very result, because the construction kit is designed for children, and the content — for high school pupils and students. But this kit suddenly began to develop a special functionality. Based on the given elements, we began to develop tasks for working in real space - to design, print images and make objects. The hybridity of this kit was unexpectedly embodied in a scenographic task using a computer monitor.

\section{Figure 6}

Virtual construction kit of gifts. CD "Return of the Magi”. 2004

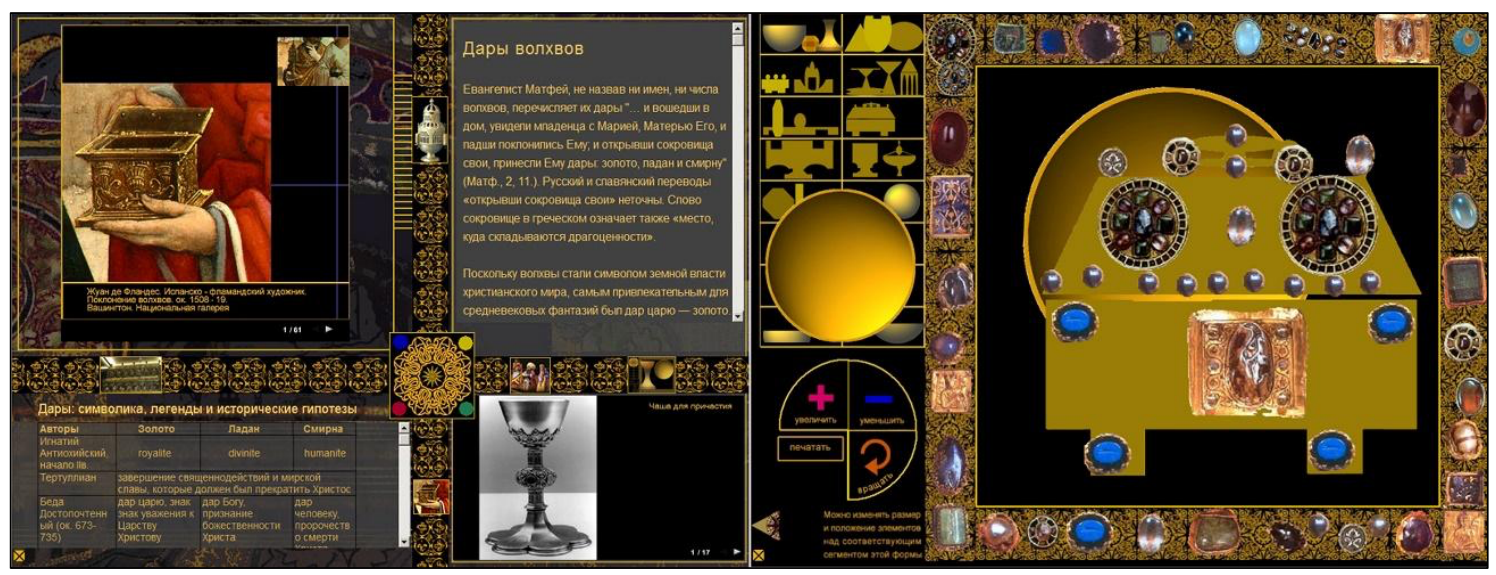




\section{Virtual construction sets for artistic propaedeutics}

An important step in the development of virtual construction kits was the project aimed at interpreting the heritage of the Russian Avant-garde by means of computer technology. It was an art educational project for teenagers, which we carried out together with the State Tretyakov Gallery. The young project participants had to develop various media projects that interpreted works from the museum collection. I suggested participants use my concept of virtual construction kits as one of the possible forms of participation. The kits created within this project have significantly expanded the understanding of the capabilities of this tool. Primarily it refers to the varied experience of designing the interfaces of these small applications. In the illustration, I show some of these works, so different and interesting in their capabilities. In these construction kits, for the first time, the goal was set to interpret the artistic heritage in the process of identifying and demonstrating the characteristics of the artist's method or artistic direction.

The most unusual in its imagery and the most difficult to create was the construction kit dedicated to the work of the architect Yakov Chernikhov. Chernikhov's graphic fantasies with skyscrapers are especially famous. To create these graphic sheets architect uses multiple repetitions of method identical lines - straight lines and ellipses. The effect of dynamic growth of structures is created, the images of colossal structures seem to be created right in front of our eyes.

This kit combines two directions of the architect's work - projects of the utopian skyscrapers (Figure 7) and fantasy on the theme of machines and mechanisms. Several types of details are used to create the mesh structures of skyscrapers, which are extracted from the already started construction. The details are also movable.

\section{Figure 7}

Virtual construction kit "Yakov Chernikhov". 2006

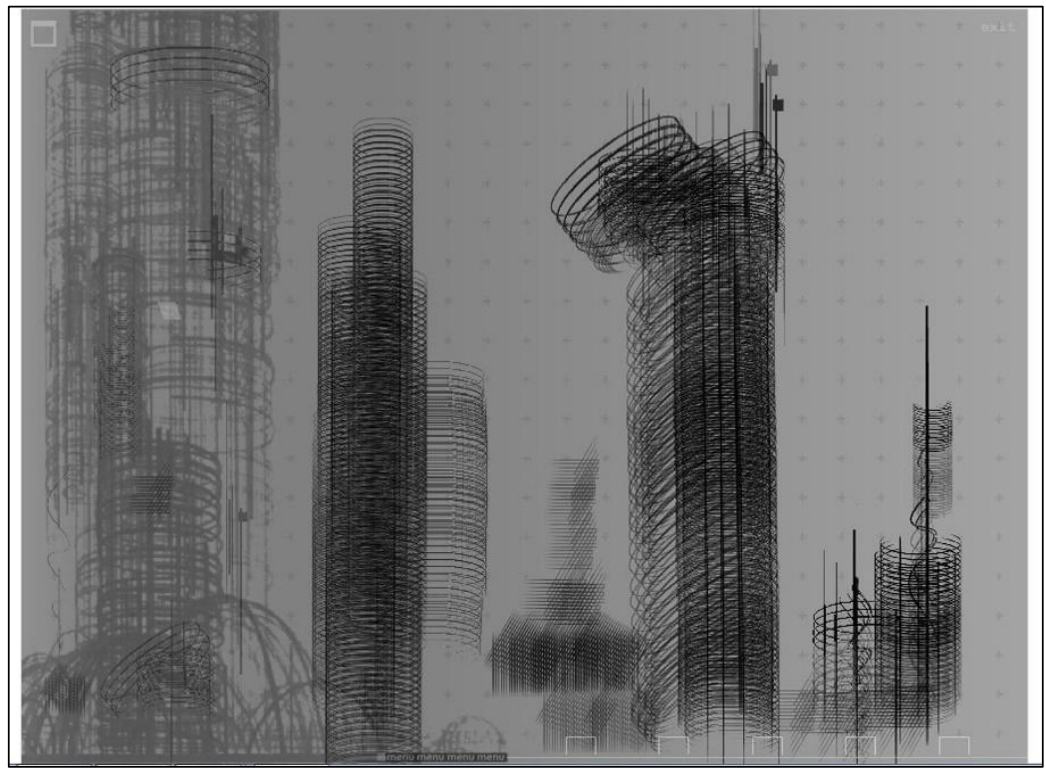

Another construction kit created as part of this project was later refined and became one of the main educational tools for our work with students. This is a kit offering acquaintance with one of the main directions of the Russian avant-garde - Suprematism (Figure 8).

At first glance, this is a fairly simple construction kit in which an image is created from a small set of geometric shapes. But the possibilities of color, tonal and compositional combinations are endless.

We use it to introduce students to the problems of formal composition, geometric abstraction, to explain the basics of the rhythmic organization of images and many other themes. This kit also has a new type of 
functionality that we have made the main standard for all future developments.

Figure 8

Virtual construction kit "Supremus". 2006-10

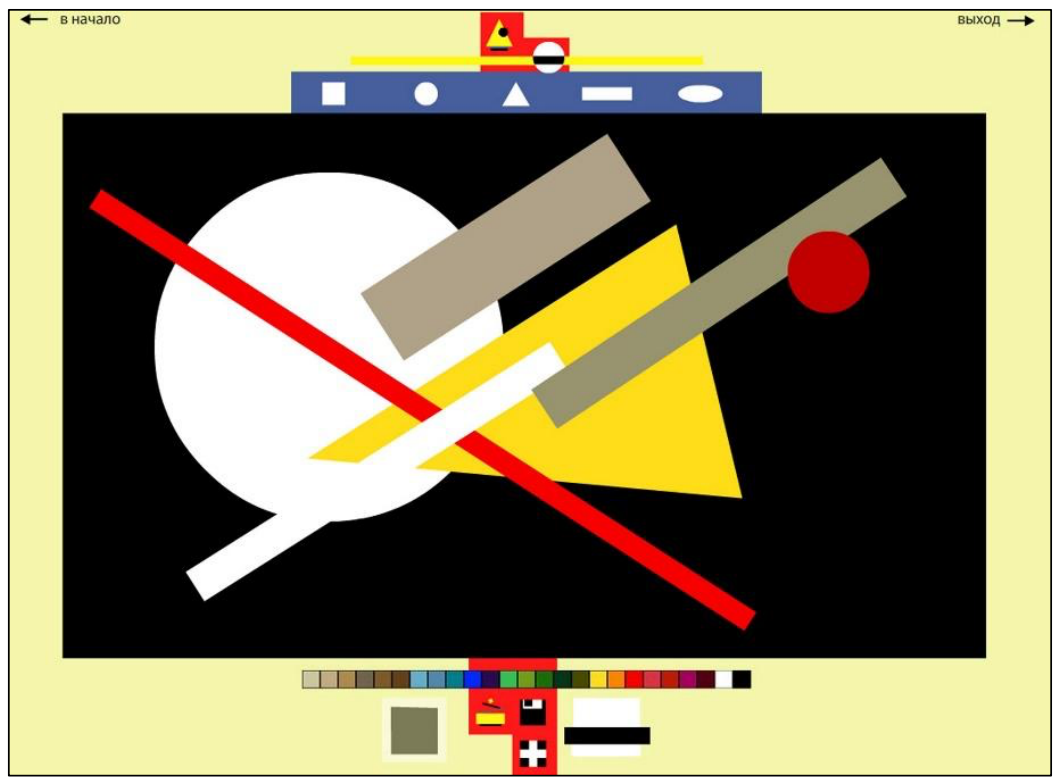

Later, this construction kit was supplemented with one more function - on-line usage, with the ability to directly publish the created works at a virtual exhibition. Another important aspect of this kit for us is a question of saving the work. The author of the work is invited to give a name to his creation, using one of the names prepared by us in advance. This is an important educational issue, which gives students an idea of the peculiarities of the artistic method of the Suprematists, who symbolize their works using industrial, technogenic metaphors. The continuation of work on the interpretation of the heritage of the Russian Avantgarde with the help of virtual kits was the work on a rather complex construction kit based on the work of the artist Vladimir Tatlin (Figure 9).

The complexity of this project lay in the fact that the artist's language was expressed with materials - iron, wood, glass and ropes. How to interpret such a pictorial language in virtual form? The selection and grouping of materials by their type was not difficult. But it became possible to express the properties of materials presented in virtual form by combining them with the material "glass," which Vladimir Tatlin used rarely. The set of glass parts in the kit and the adjustment of their transparency opened up the possibility of creating multilayer assemblies. The inclusion of glass in the composition immediately gave the impression of the authenticity of the materials, emphasizing the texture of rusty iron and rough wood. But the most important tools that made it possible to feel the specificity of the artistic language of Vladimir Tatlin were scissors and a string. Scissors is a tool using which you can cut out any silhouette. The scissors were made in such a way that you can only cut roughly - in straight lines, like scissors cut for metal. It was proposed to work on virtual assemblies at several levels, when details of the work are fixed on one of them. At the topmost, "finishing" level, it is proposed to complete the work by pulling the "string." Vladimir Tatlin designed stringed musical instruments and was very fond of introducing a stretched wire into his works. For example, in "Corner relief" (Tatlin, 1914). The string tool is only activated in the topmost layer, and setting this line feels like pulling on a string. 
Figure 9

Virtual construction kit "TATLIN". 2011

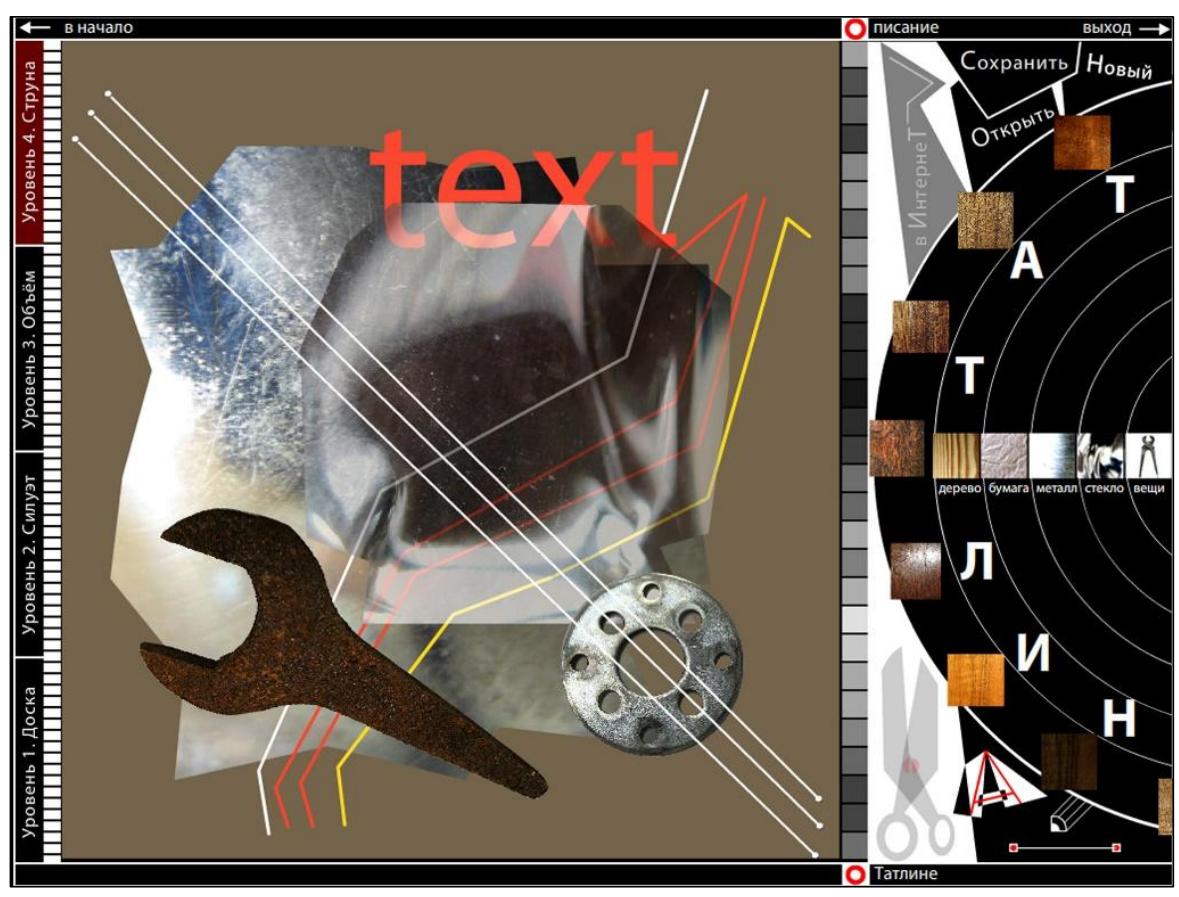

Today I think that the theme of the Russian avant-garde and constructivism will be continued in our virtual kits. The experience of the avant-garde was forcibly forgotten for a century due to the circumstances Russian art education faced. Today the enormous public interest in this heritage forms a demand for the reconstruction of the educational and methodological experience of the era of modernism. And it seems to me that this reconstruction of history should start from the beginning - with the construction kit of Friedrich Froebel. For now, this is how I understand the evolution of ideas that shaped the art culture of the 20th century and remain influential for art education in the 21 st century.

\section{Virtual construction sets for a wide audience}

Somewhat earlier than the TATLIN construction kit, we developed a semantic constructor in which the meaning of the used items was of prime importance. This kit is very different from all our other projects. It was created by my students Alexandra Selivanova and Fedor Mikhailov for fun. The construction kit is called "Robots" (Figure 10) and the user is invited to assemble his robot from various household items. But not any everyday things, but things that have become obsolete and long forgotten, which were used in the thirties and eighties of the $20^{\text {th }}$ century, and which for modern adolescents are a rarity and a wonder. The visual design of the program is reminiscent of an old radio. When the program starts, the sound of the tuned radio wave is turned on. The user is transported several decades ago, in the era of the first space flights, small-screen TVs, radio tubes. All parts in the kit are divided into groups - stationary, moving, glowing and sounding. 
Figure 10

Virtual construction kit "Robots". 2011

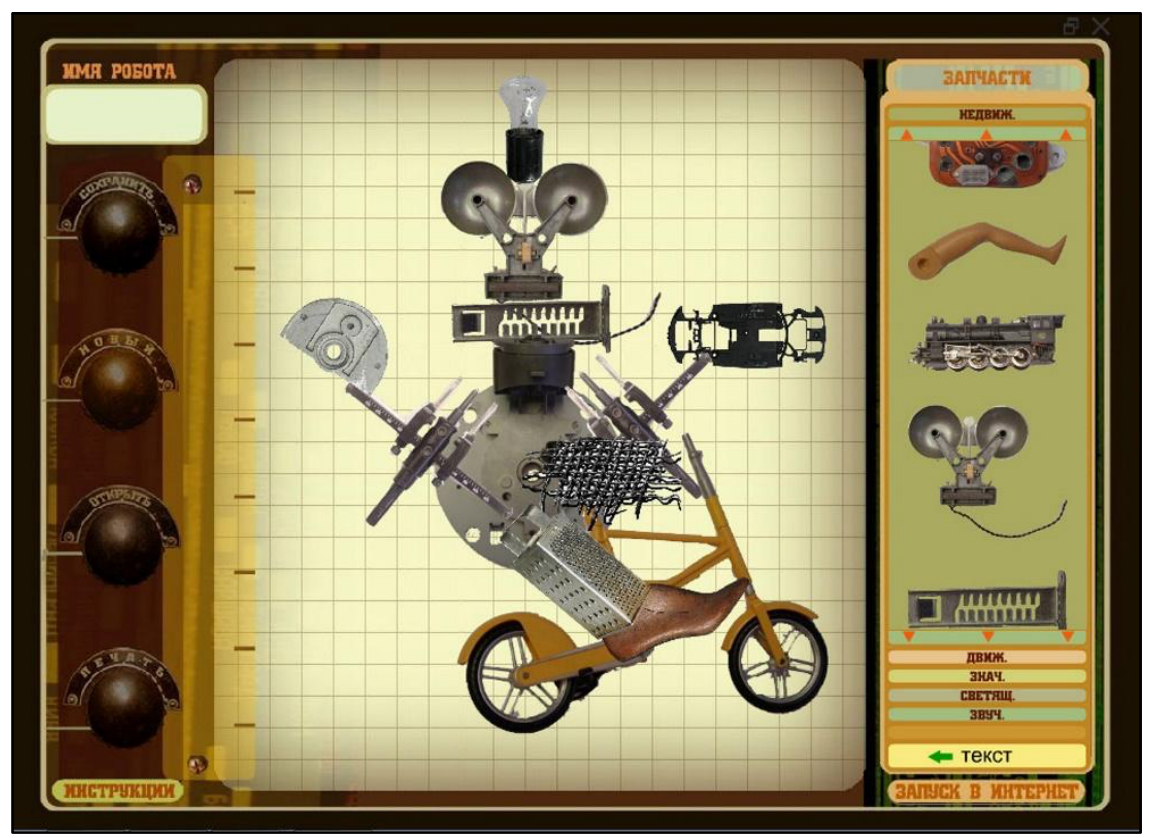

The construction kit allows you to create an audio collage from various technical noises. And from all this diversity, the author can create a unique image of a mechanical person or any device, odd, funny and very expressive. To save the work, you need to give a name to the robot, sign the name of the author. And the most interesting thing is to send the robot to an online exhibition on the Internet. Each robot is assigned a unique number by which, having switched to the exhibition screen, you can see your work among the works of other authors moving along an endless conveyor (Figure 11).

"Robots" has become one of the most popular among all our developments. This kit turned out to be interesting not only for children and adolescents, but also for adults, and is also used in family leisure time. Elderly people can explain what kind of objects they use to create their robots.

\section{Figure 11}

Virtual construction kit "Robots". On-line exhibition. 2011

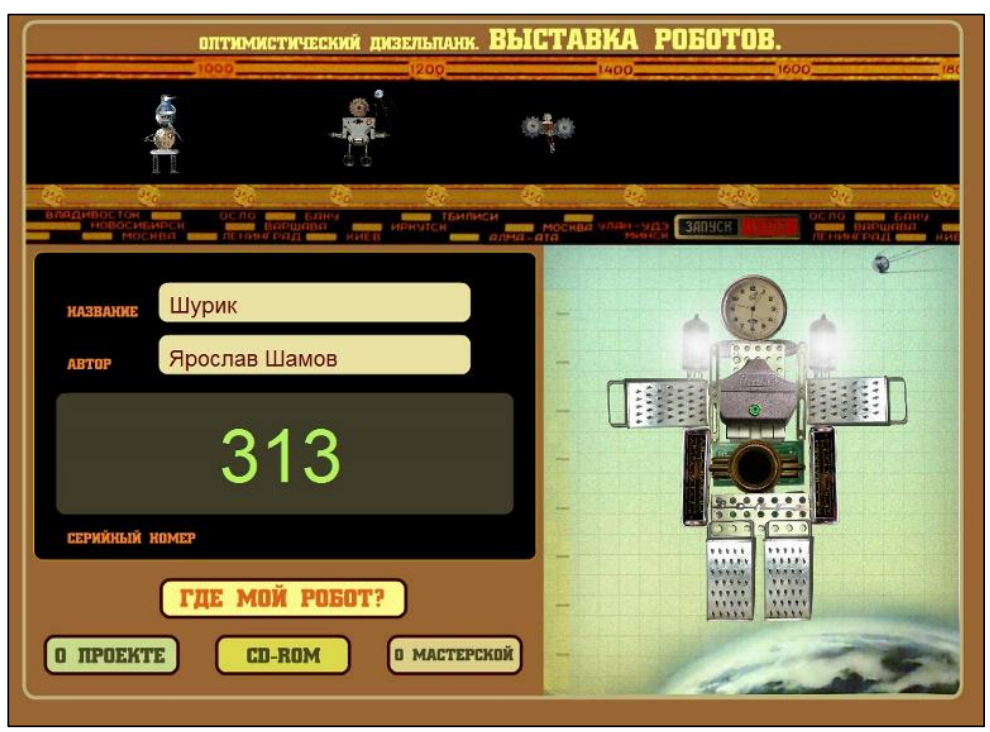


This construction kit stimulated communication between people of different ages and, even, was used as a creative leisure for professional groups. We have also considered the possibility of using this construction kit to work in real space. Each of the created images can be printed in two different forms: as a brochure cover and as a poster. The cover of the brochure is designed to create custom instructions for the created robot. Our students are happy to come up with different purposes for their robots, describe its capabilities, invent funny names for its parts. The play upon words that accompany the invention of the robot, when students compete in their wit, turns out to be very fruitful for unexpected semantic discoveries. This work exploits the natural interest of children and adolescents in old things that keep traces of time and the history of their parents. The weirdness and incomprehensibility of these old things for young users stimulates their imagination and creative energy. The project "Robots" was accompanied by a short history of steampunk, in line with the ideology of which this ironic virtual construction kit was developed.

\section{Virtual construction sets for general education schools}

The next type of construction kits was developed by me and my colleagues when one of the largest Russian publishing houses (Russkoye Slovo - Uchebnik, 2012) became interested in our developments, seeking to create a new type of visual art textbooks that would include electronic applications.

We developed three virtual construction kits that were aimed at secondary and high school students. The first one was the construction kit of fantastic creatures, based on the ancient Greek vase painting (Figure 12). The set of details consists of different fragments of the Greek vases.

The kit was called as a fantastic sea creature Hippocampus, the seahorse. Combining parts of different creatures into new images is the creative task of this constructor. Wings, tails, torsos, heads and hooves, fins - everything can be used to create your own mythological creature. But first, the author is asked to choose a vessel on which he will place his work. After choosing a vase or dish, a fragment of its surface intended for painting turns into a tablet for work. This is part of the silhouette of a vase (Figure 13).

Figure 12

Virtual construction kit "Hippocamp". 2012

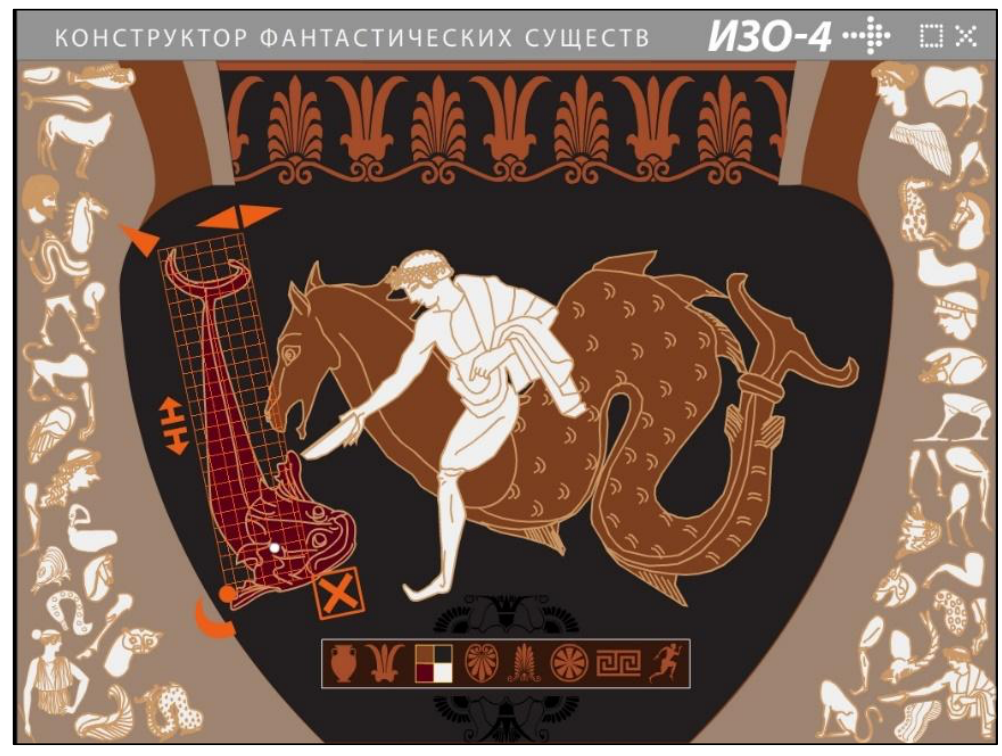


Figure 13

Virtual construction kit "Hippocamp". 2012

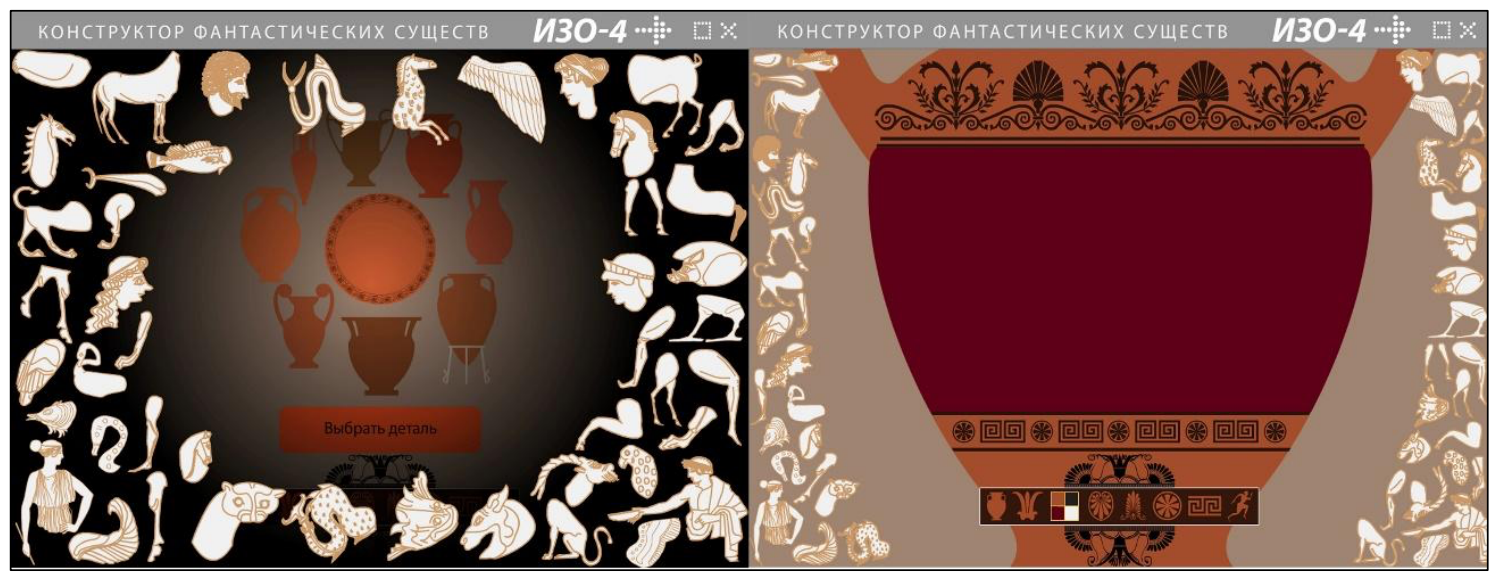

The author can choose the color on which to work. In general, all elements used in the painting can be painted in one of four possible colors - black, white, brown, purple, which corresponds to the colors of the ancient Greek vase painting. After that, construction begins. All parts are located around the working area, they can be rotated, enlarged, scaled, mirrored, connected and attached to each other. The finished work can be printed. The printed works created in this constructor are distinguished by special elegance. Also, as in the robot constructor, the new image needs to be given a name, and, following the name of the constructor itself - the Hippocampus, which consists of two parts, you need to come up with a complex name for your creature. We tried to solve another important task for all subsequent work, fundamentally replacing the instructions for using the application with a video clip. Better to see once than hear 100 times.

The second virtual construction kit for visual arts textbooks was one dedicated to the visual arts of the text. It is called Typographer (Figure 14). It helps to create different graphic compositions from letters, signs and a variety of symbols. This kit has a conceptually important function that visually explains its purpose. Here need to enter a word in the text box. When you press a button, the word scatters chaotically on the working tablet. Some letters become large, some small ones, turned in different directions. It is proposed to collect a graphic composition from these letters that could reveal the meaning of this word. This is how the futurist artists of the early 20th century scattered typography typefaces, collecting their avant-garde verses from letters of different sizes. And it was the beginning of a new era in the visual presentation of text, which laid the foundations for modern graphic design.

Among the set of elements for design in the typographer there are ancient Egyptian hieroglyphs, signs of alchemists, modern electronic pictograms, signs of electrical engineering. The color of all elements can be changed using a limited color palette. Items can be displayed, flipped, rotated and scaled. The set of elements is supplemented with a few simple geometric shapes. We have used this kit many times in our work on a variety of projects with children and teenagers.

A particularly unexpected result was the work on illustrations for the fairy tale "About Tsar Saltan" by Alexander Pushkin (Pushkin, 1968). In this task, the idea of constructing from random forms, which I formulated in my first kit from the "Artist's Book", was continued. How actively fantasy should work so that, with the help of letters and numbers, a variety of typographic signs, to depict a scene from a fairy tale in which a prince, turned into a mosquito, bites the culprit of his exile on the nose (Figure 15). But in this case, typographic signs are used as an abstract building material. In other assignments, the task was to use different typographic signs to build an expressive visual composition. The quiet work develops an understanding of the visual features of written text. And some authors want to create their own typeface or secret sign system. But projects related to the development of their own sign systems by teenagers are already beyond the boundaries of the topic of virtual construction kits. 
Figure 14

Virtual construction kit "Tipograph". 2012

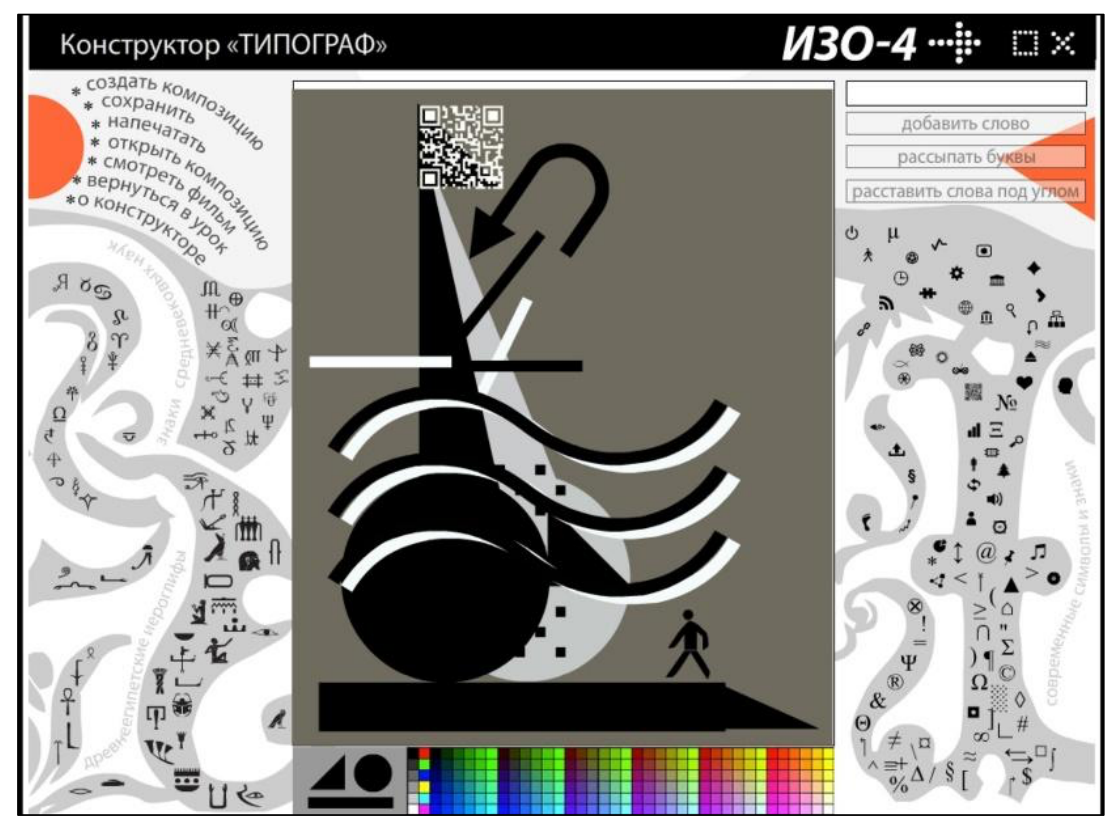

Figure 15

Virtual construction kit «Tipograph». 2012

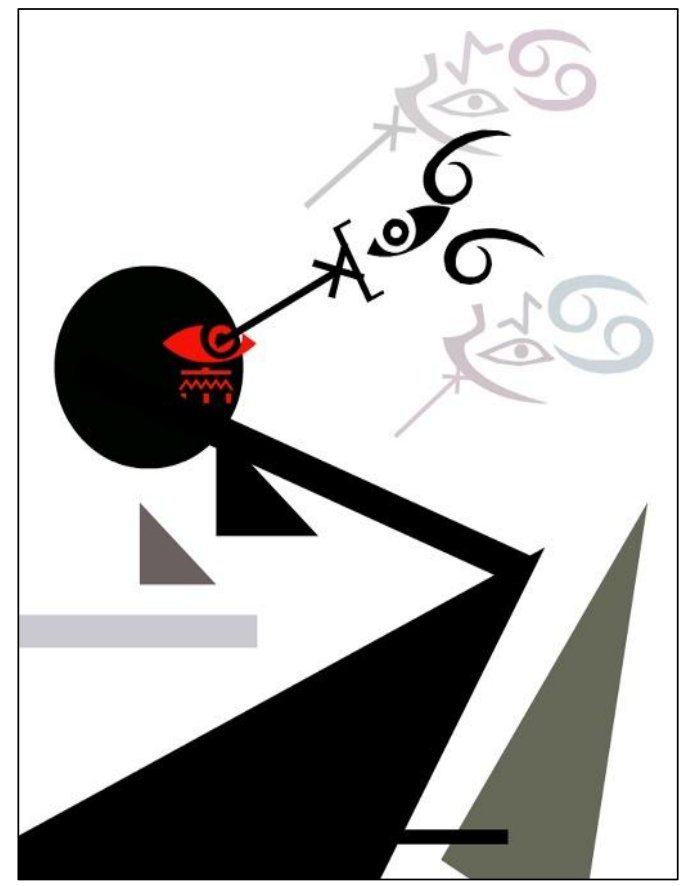


Figure 16

Virtual construction kit «Diafilm». 2012

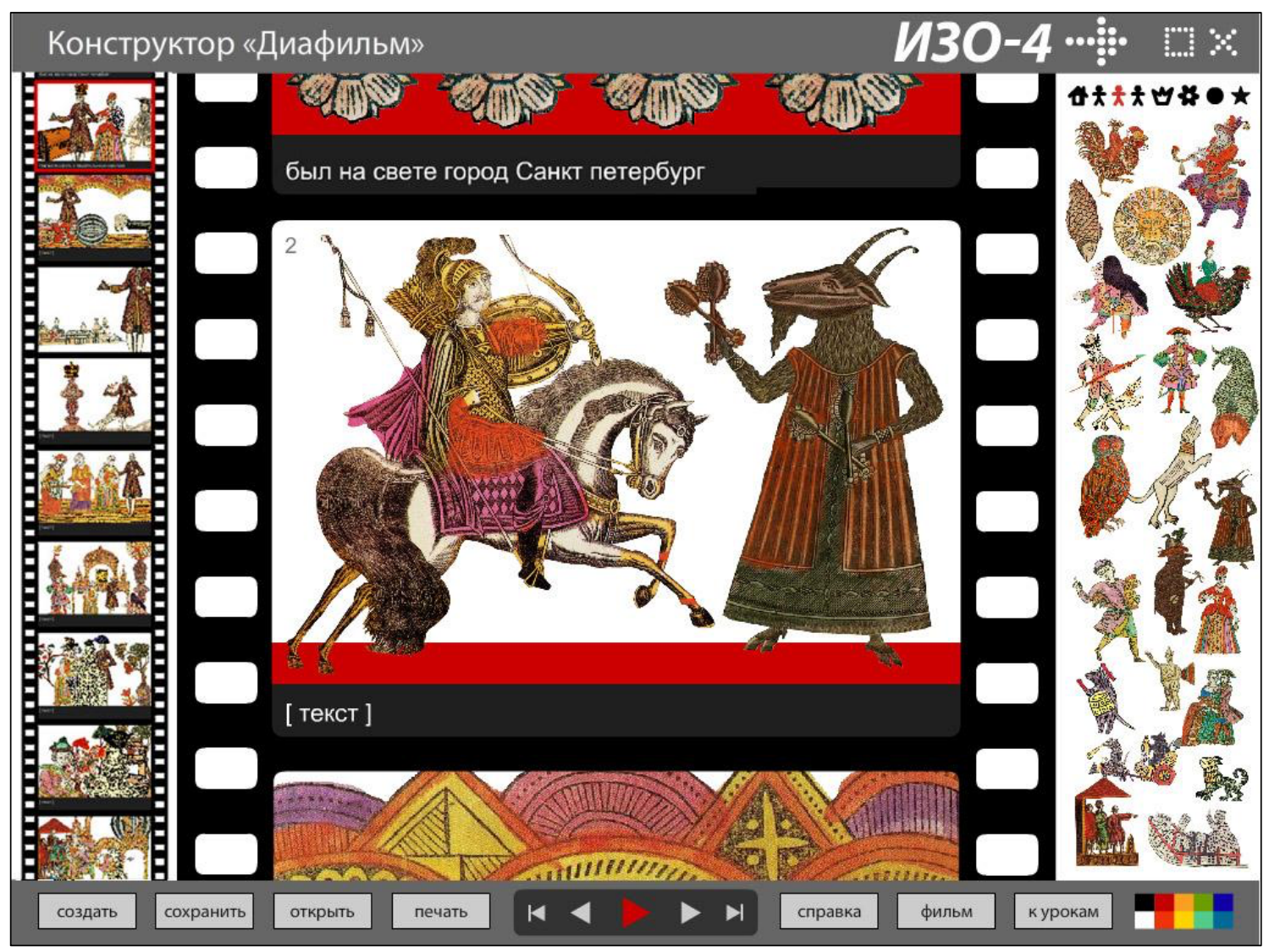

The next kit developed for the visual arts textbooks is called Filmstrip (Figure 16). It is named after the forgotten technology of frame-by-frame viewing of a film, popular in the USSR in the middle of the 20th century. Derived from the magic lantern, this technology is based on projecting an image onto a wall, accompanied by oral narration. In a film strip projector, the film is manually scrolled frame by frame. Each frame is examined, the caption is read aloud and the storyteller comments on it. The visual interface of the "Filmstrip" kit is made in a similar way. The goal of working with the kit is to create a short story consisting of 12 frames. Each frame is filled with characters and different objects and must be supplemented with a signature. By pressing the scroll key to change frames, the child sees the smooth movement of the perforated film. An unexpected decision of choosing the details was made by the designer of the kit. To express the antiquity of the tradition of creating visual narratives, fragments of Russian folk splints pictures are used as details for construction. These folk pictures, funny and ridiculous, with fairy-tale characters and animals were brightly painted by hand. Thanks to the brightness of color and expressiveness of elements for construction, "Filmstrip" looks very decorative and cheerful. This positiveness Is transmitted to all the stories that are composed with the help of the kit. Naturally, among the works created, most are fairy-tale subjects. But there are very curious attempts by young authors to tell completely different stories, endowing the characters of folk pictures with functions that are not peculiar to them. There are examples of creating detective stories about Sherlock Holmes, descriptions of military adventures and travels. These experiences show a strong desire among children to tell their own story with a bright and inspiring instrument. You can print the entire feed created in the Filmstrip Designer by turning the screen story into a small book with a cover. "Filmstrip" allowed us to carry out project activities with children, using only a virtual construction kit, because creating a story, even a small one, is a whole project. A large number of different virtual kits help us sometimes to conduct public workshops in museums. Teenagers work with interest, but gradually, in the process of work, more and more visitors switch to the most popular ones - 
these are the "Robots" kit and the "Imagine a landscape" one.

\section{Virtual construction sets in museum activities}

"Imagine a landscape. Virtual construction kit based on the works of Fyodor Matveev" is the full name of the most successful and popular kits we have created (Figure 17). This kit was created specifically for the exhibition of a Russian painter of the early 19th century. The work of Fyodor Matveev fully fits into the historical boundaries of classicism painting, created within the famous canon - four plans of the picturesque space, the so-called "backstage", staffage. Following this clearly defined system of organizing the pictorial space, we have developed a virtual construction kit that allows you to feel like a master of a classic landscape. The main issue in a kit is a set of elements for construction. The set consists of eight sections with fragments of Matveev's paintings - Architecture, Plants, Clouds, Mountains, Earth, Water, Backstage, Staffage. There is also a ninth section with graphics from the 19th century.

\section{Figure 17}

Virtual construction kit «Imagine a landscape». 2008, 2017

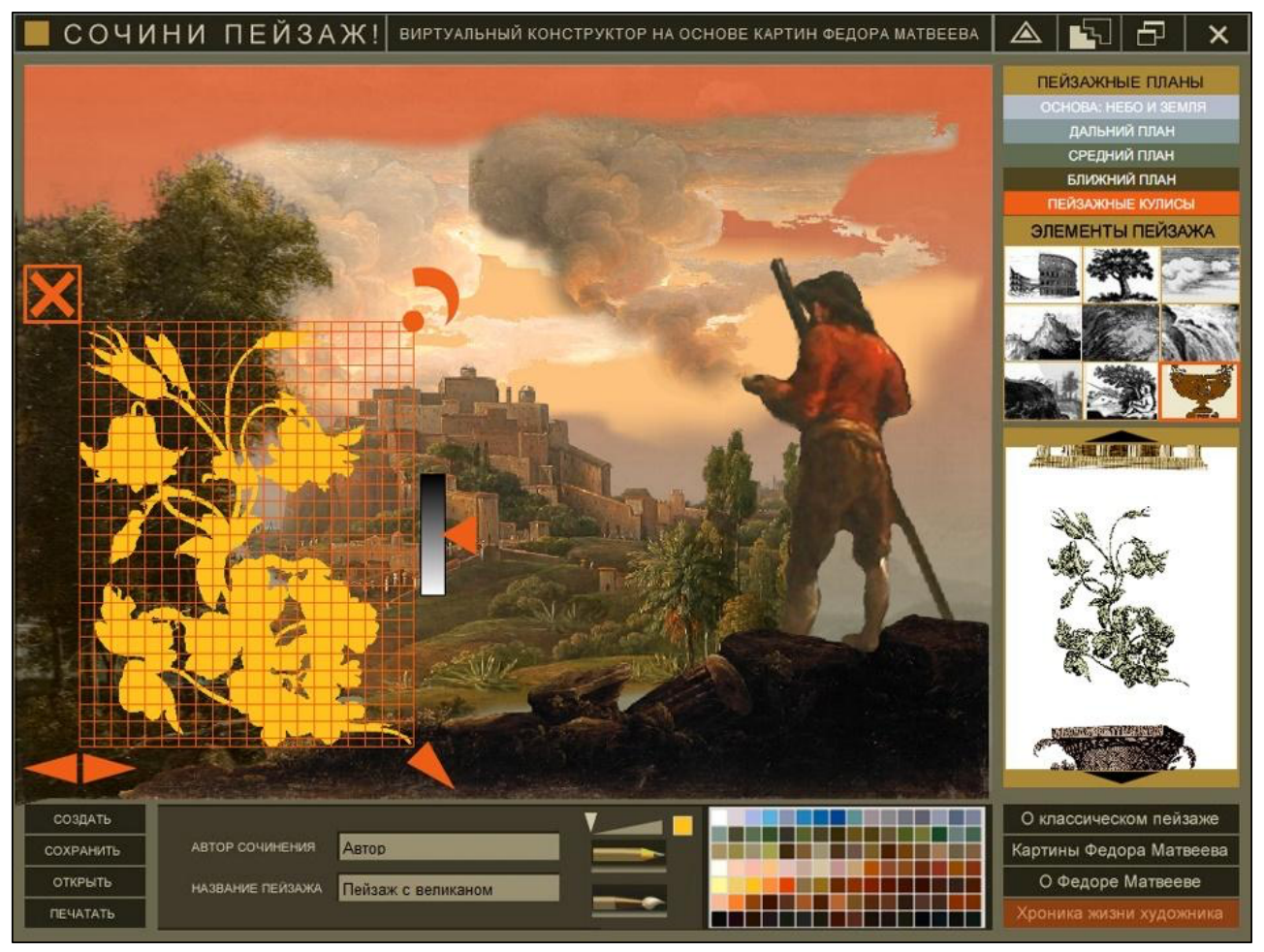

The work begins with setting the horizon line, which can be raised and lowered. The horizon line sets the proportional relationship between the earth and sky. The earth and sky can be illuminated using a palette of selected colors. Then we are ready to move on to the very spatial plans of painting of classicism - there are only four of them. In the background we set blue and lilac mountains, golden clouds. They cannot be edited while on other planes. Then we begin to collect the landscape, establishing hills and waterfalls, ancient temples and copses. We place trees in the foreground, creating a whimsical composition and staffages - figures of people and animals, giving a sense of scale. We complete the construction of the image with the help of dark "wings" of dense thickets and bushes - left and right. The wings create the illusion of depth in space. Here, in the foreground, you can install a vase, column or capital from a set of additional parts. In this process of assembling a landscape and joining parts from different paintings by Fyodor Matveev, we will really need a tool for working with tone. Chiaroscuro is the foundation for creating aerial perspective and for connecting parts. With this tool, you can change the tone of the selected part from white to black. After completing work on the landscape, the result can be framed, as artists of the past did, and then printed. 
The toolbox also contains a palette with the colors of Fyodor Matveev's painting, which you can use to fill details with color (Figure 18). If desired, any detail turns into a colored silhouette. And this simple tool works like a time machine that transports us to the present day. The place of tonal harmony - chiaroscuro, is taken by the main method of modern visuality - collage (Figure 19).

Figure 18

Virtual construction kit «Imagine a landscape». 2008, 2017

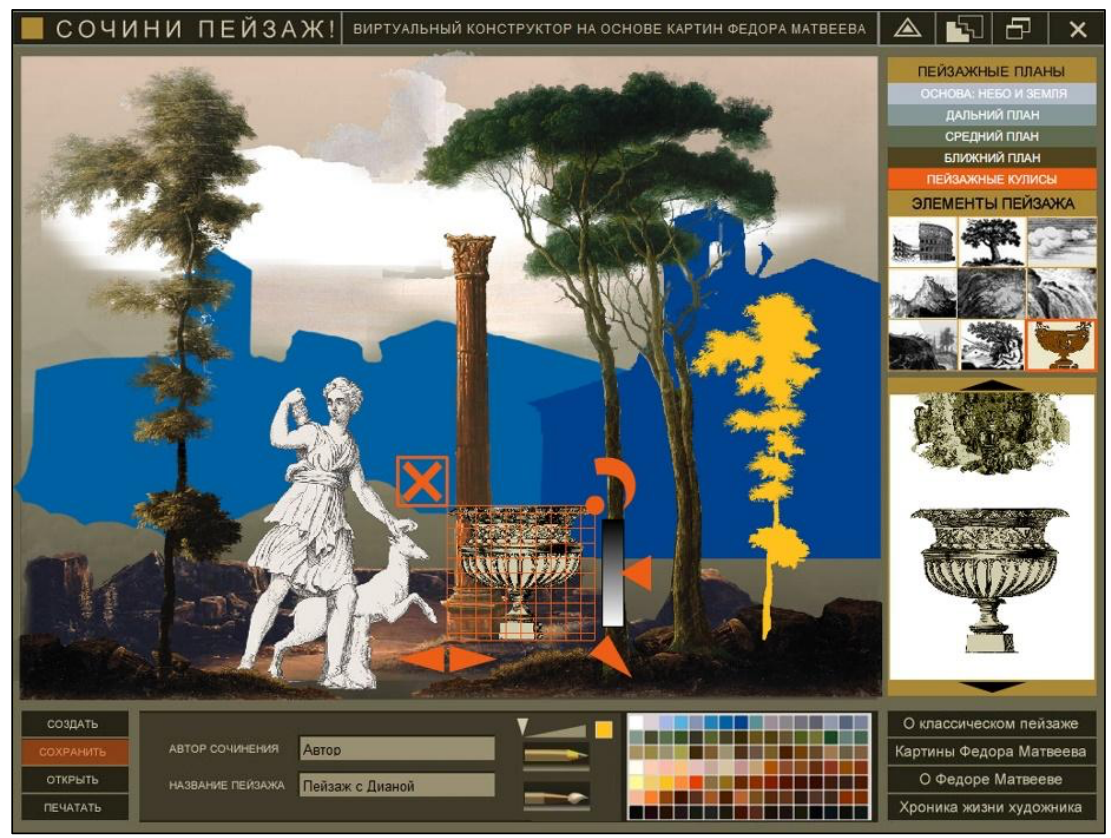

This feature motivates the user to experiment and play in the space between the art of the past and the present. Speaking about the "Imagine a Landscape" kit, I would like to raise the issue of the semantic context necessary for working with construction kits. This problem has two aspects: a description of kit's capabilities and a description of the phenomenon to which this kit is dedicated. We solved the problem of describing tools and opportunities for work by creating video clips built into each kit. I have mentioned this above. We have added a function to automatically start the movie when there is a long pause in work. When used in exhibitions and public workshops, this feature is very useful, making our kits more spectacular.

The second task - revealing the context of the phenomenon to which the construction kit is dedicated - is much more difficult to solve. Moreover, the optimal solution to this problem is associated with the use of virtual construction kits in the context of educational content. This is how the visual arts textbooks were designed. Construction kits from the "Artist's Book," construction kit "Robots," construction kit «Imagine a landscape" can be used without intermediaries. In the Artist's Book, the appearance of construction kits is preceded by a large theoretical material - several chapters devoted to the history of visual communication. The construction kit "Robots" is clear enough to use and comprehend the phenomenon to which it is dedicated. A small article about dystopias and steampunk complements this construction kit, but in principle it is superfluous for work. The construction kit "Imagine a Landscape" directly represents the artist's work, including a complete electronic catalog of his works. The work with the kit is preceded by a small chapter describing the canon of classicism painting, accompanied by several schemes. However, the problem of using virtual construction kits without an adult middleman remains challenging. While using a virtual construction kit without an intermediary, it is necessary to create detailed educational material, which will turn into the very necessary semantic context. Such an approach is closer to me. Although small and very simple kits, placed, for example, in the context of the exhibition and existing only within its limits, significantly expand the information capabilities of any project. When developing virtual constructors, I strive for technological simplicity. 


\section{Figure 19}

Virtual construction kit «Imagine a landscape». 2008, 2017

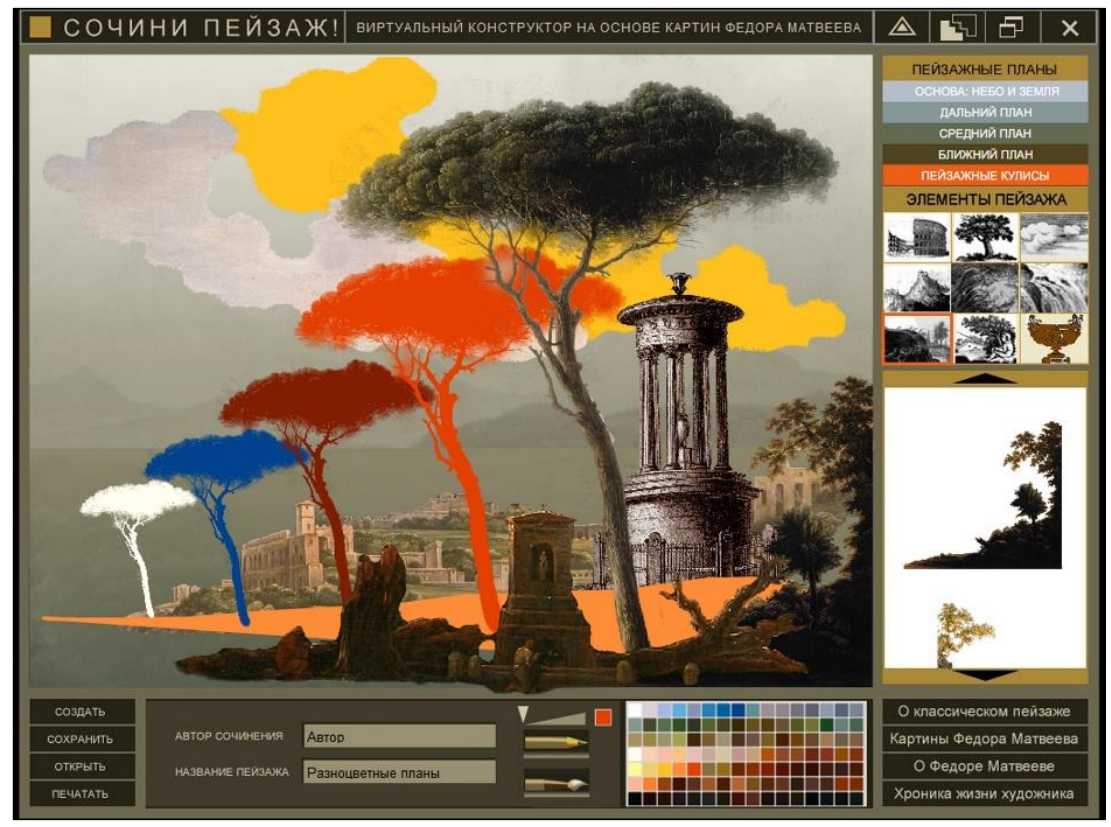

Construction kits are like wet sand boxes, ancient drawing tools. On the surface of the sand, you can easily create images with a stick, then level the sand and again on a flat surface continue to come up with new images. The most interesting thing about virtual kits is playing with visual elements. More precisely, these are game actions, free manipulations with the details. As a result of these actions, two new entities appear - a visual image and an idea of an artistic phenomenon, to which each construction kit is dedicated. It creates new content and assimilates the experience of culture, combining everything into a single process of cognitive play.

Virtual construction kits are part of a more general idea that any construction kit is a tool for creating new models, individual art projects. After all, each artist creates their own unique kit, consisting of a set of elements developed by him and methods of assembling these elements into a whole work. For the loggia's paintings in Vatican, Raphael developed a "grotesque" construction kit based on archaeological findings the paintings of the Golden House of Emperor Nero. Eugene Delacroix developed color systems for his paintings, the so-called plaques. Giorgio Morandi painted bottles in the colors he needed, which he used for many years for his still lifes. Rembrandt, Picasso, Joseph Beuys, Malevich, and El Lissitzky had their own construction kits - in general, all artists who create a unique author's project. It is impossible to create a unique style in art without developing your own exceptional construction kit. While creating virtual construction kits, I think about possible reconstructions, interpretations of such artistic construction kits. It seems to me that today it is very important to find new ways to understand the artistic heritage and to open up new approaches to the perception of art.

\section{Artist's book (2004)}

\section{Characteristics of virtual construction set examples}

The thematic context for the "Artist's book" system is the global change of the main medium of information when the digital environment comes to replace book printing. The goals of the "Artist's book" are aimed at the formation of ideas about sign-symbolic construction and hybrid forms of information representation, uniting different media.

The "Artist's book" system is aimed at adolescents interested in contemporary art and design, students of art colleges, and art departments of universities. The recommended age is 12 years and older. 


\section{Return of the Magi (2004)}

The thematic context of the educational system "Return of the Magi" is the development of European imagery, late antiquity, Christian ritual.

The goals of "Return of the Magi" are aimed at forming an idea of the evolution of the sign-symbolic content of Christian ritual from late antiquity to the Renaissance.

Return of the Magi can be used by two different age groups. Elementary school pupils can use only a construction set. Students of high school, colleges, and university art departments first master the historical and symbolic context, after which they experiment with a virtual construction set. The recommended age is 8 years and older.

\section{Yakov Chernikhov (2006)}

The thematic context of the virtual construction set "Yakov Chernikhov» is the utopian project of the Russian Avant-garde and paper architecture.

The construction set introduces the imagery of industrial culture, forming an idea of the author's visual technology.

The construction set is intended for secondary and high school students, as well as for specialized art studios and art schools. Recommended age is 14 and older.

\section{Supremus (2006, 2010)}

The thematic context of the virtual constructor Supremus is the imagery, symbolic system, and pictorial methods of Suprematism. The construction set aims to form basic concepts of visual composition, propaedeutics of the Russian Avant-garde. "Supremus" introduces the art of Suprematism to high school students and is used to learn the basics of composition in specialized art studios and art schools. The recommended age is 12 years and older.

\section{TATLIN (2011)}

The thematic context of the virtual construction set "TATLIN" is the art of Constructivism. The construction set forms an idea of the meaning of material and construction as the basis of the semantic and figurative structure of a work of art. "TATLIN" construction set is aimed at secondary and high school students, used to teach the basics of composition and design theory in art schools. Recommended age is 14 years and older.

\section{Robots (2011)}

The thematic context of the virtual construction set "Robots" is steampunk, pop art, post-industrial art of the late 20th century. The goals of "Robots" are the formation of an idea of irony as a means of semantic construction of art objects, the symbolization of old household items, the experience of constructing from waste. The "Robots" construction set can be used by a wide range of members, for joint activities of parents with children, for classes with children with limited mobility. The recommended age is 7 years and older.

\section{Hippocamp (2013, 2015)}

The thematic context of the virtual constructor "Hippocamp" is the ancient Greek vase painting, the mythology of Ancient Greece, polymorphic mythological creatures. The construction set is aimed at creating images of new polymorphic creatures and their symbolization in the context of ancient Greek mythology. Constructor "Hippocamp" is designed for the visual arts in elementary school. Besides, the construction set is used in history and literature classes in secondary and high school. Recommended age is 9 years and older.

\section{Tipograph $(2013,2015)$}

The thematic context of the virtual constructor "Tipograph" is the history of writing, sign systems, infographics, and typography.

The construction set forms an idea of the sign as a visual object, the construction of graphic compositions from pictograms introduces the basics of graphic design. The "Tipograph" construction set is designed for visual arts classes in high school, as well as for classes in the basics of composition in art schools. The recommended age is 10 years and older. 


\section{Diafilm (2013, 2015)}

The thematic context of the virtual construction set "Diafilm" is an oral culture, narrative art forms, popular prints, comics, magic lantern. It develops skills in creating visual stories, organizing the composition of each screen, and projecting an image of the entire sequence of frames. The construction set "Diafilm" is designed for the visual arts in primary and secondary schools. Also, the construction set is used in literature classes in middle and high school. The recommended age is 7 years and older.

\section{Imagine a landscape $(2008,2017)$}

The thematic context of the virtual construction set "Imagine a landscape" is a painting of the era of classicism, the problem of the canon, and innovation in art.

The construction set forms an idea of the methods of organizing landscape images that had developed in European painting by the beginning of the 19th century.

"Imagine a landscape" was intended to be undertaken in the halls of the museum during the exhibition of the artist Fyodor Matveyev (State Tretyakov Gallery, 2008). After the exhibition, the constructor is used in different cultural contexts for edutainment programs. Recommended age is 9 years and older.

\section{References}

Brosterman, N. (1997). Inventing Kindergarten. Harry N. Abrams.

Buhler, K. (1924). Spiritual development of the child (V. Smirnov, Trans.). Novaya Moskva.

Korzybski, A. (1995). Science and Sanity: An Introduction to Non-Aristotelian Systems and General Semantics. Institute of General Semantics.

Pushkin, A. S. (1968). The tale of Tsar Saltan, his glorious and mighty hero, Prince Guidon Saltanovich, and the beautiful princess Swan (Vol. 3). Khudozhestvennaya literatura.

Savenkova, I. G., Ermolinskaya, E. A., Selivanova, T. V., Selivanov, N. L. (2012). Art. 1-8 grades (Vol. 12). Russkoye Slovo - Uchebnik.

Vygotsky, L. S. (1984). Tool and symbol in child development (Vol. 6). Pedagogika.

\section{APA citation format $\left(7^{\text {th }}\right.$ edition) for this publication:}

Selivanov, N. (2021). Virtual construction kits. In J. Lee, S. M. Christensen, S. Beene, X. Chen, and W. Huang (Eds.), Visual literacy in the virtual realm: The book of selected readings 2021 (pp. 94-111). International Visual Literacy Association. https://doi.org/10.52917/ivlatbsr.2021.019 